EPJ Web of Conferences 116, 06011 (2016)

DOI: $10.1051 /$ epjconf/201611606011

(C) Owned by the authors, published by EDP Sciences, 2016

\title{
Measuring the optical properties of IceCube drill holes
}

\author{
Martin Rongen ${ }^{\mathrm{a}}$ for the IceCube collaboration ${ }^{\mathrm{b}}$ \\ III. Physikalisches Institut B, RWTH Aachen University, 52074 Aachen, Germany
}

\begin{abstract}
The IceCube Neutrino Observatory consists of 5160 digital optical modules (DOMs) in a cubic kilometer of deep ice below the South Pole. The DOMs record the Cherenkov light from charged particles interacting in the ice. A good understanding of the optical properties of the ice is crucial to the quality of the event reconstruction. While the optical properties of the undisturbed ice are well understood, the properties of the refrozen drill holes still pose a challenge. A new data-acquisition and analysis approach using light originating from LEDs within one DOM detected by the photomultiplier of the same DOM will be described. This method allows us to explore the scattering length in the immediate vicinity of the considered DOMs.
\end{abstract}

\section{Motivation}

The IceCube Neutrino Observatory [1] is situated in the deep glacial ice below the geographic South Pole. The IceCube sensors are 5160 digital optical modules (DOMs) which detect Cherenkov light produced by relativistic charged particles traversing the detector. Each DOM incorporates a 10 inch downward facing photomultiplier as well as several LED light sources for calibration [2] (see Fig. 1). The DOMs were deployed in a depth between $1450 \mathrm{~m}$ and $2450 \mathrm{~m}$ in 86 holes where the ice had been melted with a hot water drill [3].

The deep Antarctic ice has long been known to have exceptional optical properties, as trapped air bubbles shrink and eventually vanish under large pressure forming an air clathrate hydrate [4]. For accurate reconstruction of the properties of traversing relativistic particles, a precise knowledge of the absorption and scattering lengths of the ice traversed by the Cherenkov photons is crucial. The vertical structure of the undisturbed glacial ice or "bulk ice" was originally measured by so called dust-loggers [5], which were lowered into the drill holes. A small fraction of the light from a horizontal laser beam was redirected by scattering centers in the ice, mainly dust particles, to a downward facing PMT optically separated from the light source. In addition, data from flashing LEDs of known brightness situated in each DOM has been used to derive an accurate model of the optical absorption and scattering in the bulk ice [6].

\footnotetext{
a e-mail: martin.rongen@icecube.wisc.edu

${ }^{b}$ https://icecube.wisc.edu/collaboration/authors/2015/09
}

This is an Open Access article distributed under the terms of the Creative Commons Attribution License 4.0, which permits unrestricted use, distribution, and reproduction in any medium, provided the original work is properly cited. 


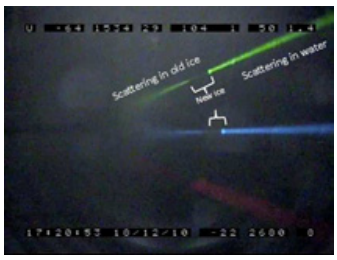

(a) Camera looking side- (b) ways into the bulk ice

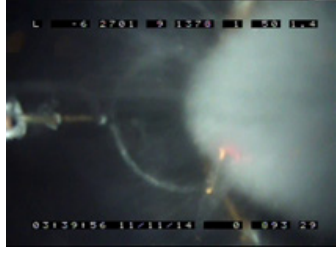

(b) Camera looking downward into the hole ice

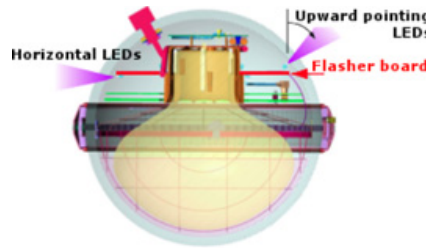

(a) Location of flasher LEDs

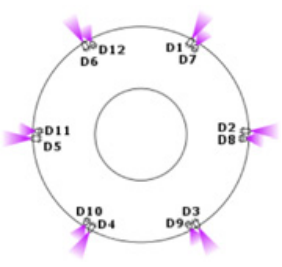

(b) LED numbering

Figure 1. The hole ice as seen by the camera.

Figure 2. Schematic overview of an IceCube DOM.

While the dominant part of the propagation of Cherenkov photons happens in the bulk ice, each photon detected by a DOM also has to propagate through the refrozen water in the drill holes, called "hole ice". The optical properties of the hole ice are less understood than the bulk ice and present one of the largest uncertainties to neutrino oscillation measurements in IceCube [7]. This paper presents a new method to determine the scattering length in the hole ice.

\subsection{Previous hole ice measurements}

The hole ice was directly imaged by a camera installed at the bottom of a drill hole in the center of IceCube [8]. It suggests two hole ice components: A clear outer region (Fig. 1(a)) and a central column of $\sim 8 \mathrm{~cm}$ diameter with a very small scattering length (Fig. 1(b)). This observation is consistent with cylindrical freezing, where impurities or air bubbles are pushed along the freezing boundaries until they merge in the center. A quantitative evaluation of the the hole ice was first conducted with IceCube's predecessor AMANDA [9] by measuring the arrival time distribution of photons from flasher LEDs with optical modules (OMs) in adjacent holes [10]. The distributions were compared to simulations assuming scattering on spherical air bubbles, resulting in an effective scattering length between $100 \mathrm{~cm}$ and $350 \mathrm{~cm}$. The effective scattering length $l_{\text {eff }}$ is related to the mean geometric scattering length $l_{\text {geo }}$, the mean distance between two scattering processes, by the mean of the scattering function:

$$
l_{\text {eff }}=l_{\text {geom }} /(1-\langle\cos \theta\rangle) .
$$

This result was incorporated into the AMANDA detector simulation as a modification of the angular acceptance function of the OMs with respect to the lab measurement (see Fig. 5) and is also used by IceCube as the default hole ice model [6]. In this model the acceptance in the forward direction of the PMT is reduced with respect to the lab measurements as the hole ice scatters away incident photons, while the acceptance in the backward direction is increased as photons have a chance to be scattered to the front of the DOM. Since then, multiple AMANDA and IceCube studies using inter-string LED data $[11,12]$ or muon data $[13,14]$ have qualitatively broadened our understanding of the hole ice, however arriving at partially conflicting angular acceptance functions.

\section{Same-DOM flasher measurements}

For the determination of hole ice properties it is unfavorable to measure the light emitted by one DOM with a another DOM, as the bulk ice properties will dominate the propagation. Each DOM possesses twelve $405 \mathrm{~nm}$ LEDs situated on a dedicated board above the DOM mainboard (Fig. 1). The LEDs are organized in six pairs $60^{\circ}$ apart. One LED of each pair shines into the ice horizontally and one LED is angled $\sim 45^{\circ}$ upward. The LEDs can be controlled individually. The dimmest stable pulse contains $\sim 257 \cdot 10^{6}$ photons in a $\sim 30 \mathrm{~ns}$ long pulse. By turning on the LEDs sequentially and measuring the 


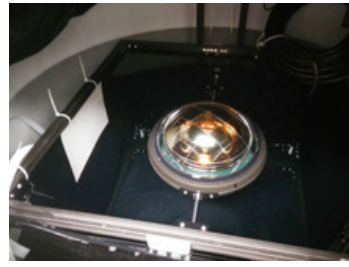

(a) DOM in water tank

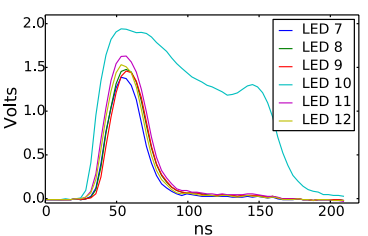

(b) PMT waveforms
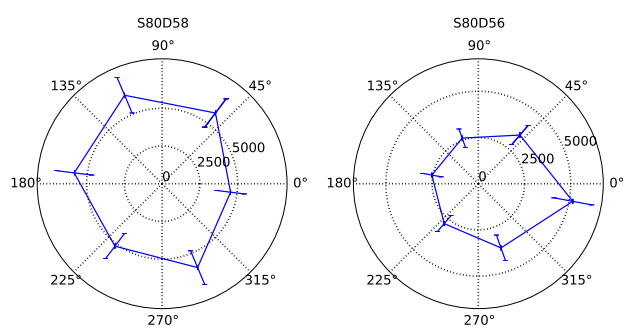

Figure 4. Typical azimuthal patterns of detected PEs on two in-situ DOMs, ranging from $\sim 2000$ to $~ 7000$ PE.

Figure 3. Lab demonstration of the measurement principle.

\begin{abstract}
(⿸丆口
\end{abstract}
intensity of light returning to the PMT of the same DOM a measurement similar to the dust logger can be performed, where the measured intensity is proportional to the scattering coefficient in the direction of the LED. This method was demonstrated by conducting the described procedure with a DOM in a water tank with an absorptive black lining, which contained a reflective sheet on one side (Fig. 3(a)). Figure 3(b) shows the average recorded PMT waveforms for each tilted LED. The LED pointing in the direction of the reflective sheet (LED 10) yields the strongest PMT signal.

\title{
2.1 DARD: Data acquisition for a flasheR DOM
}

The DOM firmware used for standard data acquisition (DAQ) [15] disables the high voltage to the PMT while the flasher board is enabled. Thus, a custom DAQ based on a debugging firmware had to be developed. The resulting system controls a single IceCube string, while the rest of the detector remains in normal operation. After setting up the high voltage to a gain of $\sim 10^{5}, 100$ waveforms are recorded for each LED configuration. This procedure has been applied to a total of 20 DOMs in two runs.

\subsection{Photon counting}

The raw waveforms are averaged, corrected for the digitizer pedestals and then calibrated [1]. The integral over the resulting waveform voltage bins $\left(V_{i}\right)$ is directly proportional to the number of recorded photoelectrons (PEs), where $Z$ is the frontend impedance and $f$ the sampling frequency of the digitizers:

$$
\mathrm{PE}_{\exp }=\frac{1}{Z(\Omega)} \cdot \frac{1}{e} \cdot \frac{1}{\mathrm{f}(\mathrm{Hz})} \cdot \sum_{i} V_{i} \cdot \frac{1}{\text { gain }} .
$$

Two examples of the number of measured PE as a function of the azimuthal LED direction can be seen in Fig. 3. Note, that light output varies by about $20 \%$ between different LEDs.

For this study only information from the tilted LEDs is used, as the horizontal LEDs showed nonnegligible saturation effects and are possibly subject to internal light contamination. Where a small fraction of the photons can reach the PMT before leaving the DOM.

\section{Simulation}

A detailed Geant 4 model of a DOM has been placed in the center of a $60 \mathrm{~cm}$ diameter hole ice column surrounded by a $600 \mathrm{~m}$ bulk ice volume. For both media the Henyey-Greenstein scattering function [16], which is a good approximation of Mie scattering and which is also used in the analysis of the bulk ice, is assumed with an average deflection angle $\langle\cos (\theta)\rangle=0.9$. The different assumption regarding the scattering process compared to [10] implies that impurities in the hole ice dominate the light scattering 

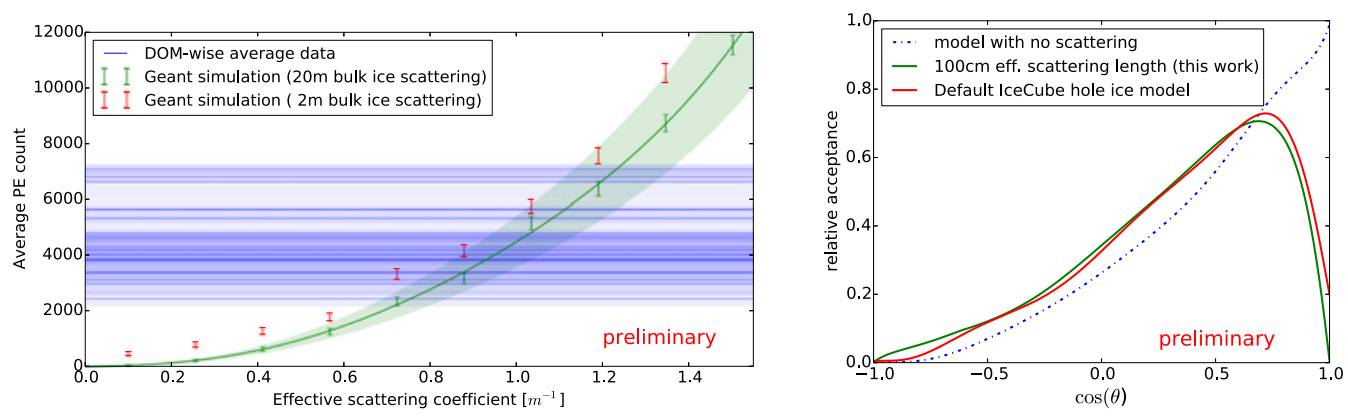

Figure 5. Comparison of the experimental and simulated light intensity arriving at the PMT of the emitting DOM.

Figure 6. Comparison of angular acceptance models derived from lab measurements without scattering and modified for the hole ice properties.

instead of air bubbles. The number of PEs detected by the DOM $\left(\mathrm{N}_{\mathrm{hits}}\right)$ is then given by the amount of photons arriving at the photocathode times the quantum efficiency and mean charge:

$$
\mathrm{PE}_{\mathrm{sim}}=\mathrm{N}_{\text {hits }} \cdot 0.25 \cdot 0.85[\mathrm{PE}] \text {. }
$$

The primary systematic uncertainty involved in this simulation is a $20 \%$ uncertainty on the absolute photon output of the flasher LEDs. Currently not considered systematics include absorption in the hole ice, the collection efficiency of the PMT at low voltages, shadowing by the DOM cable as well as positional uncertainties of the LEDs. The simulation has been performed for two extreme values of the geometric scattering length of the bulk ice of $20 \mathrm{~m}$ and $2 \mathrm{~m}$ and a wide range of effective scattering coefficients of the hole ice column as shown in Fig. 5. The results stated below are for a bulk ice of $20 \mathrm{~m}$ scattering length that applies to most of the selected DOMs.

\section{Extracting ice parameters}

The measured number of returning photons does not show a strong dependence on the azimuthal flasher direction for any DOM. A strong inhomogeneity of the hole ice, such as small column that encompasses some but not all of the flashers, is not supported by the current data. In order to quantify the average hole ice properties, the number of PEs observed from the different LEDs have been averaged for each DOM. The distribution of measured PEs compared to the simulated expectation for different scattering coefficients is shown in Fig. 5. The scattering coefficient at which the simulated number of PEs crosses the measured PE count describes the mean properties of the hole ice around the specific DOM (for the assumptions used in the simulation). The systematic uncertainty of the simulation is denoted by the green shaded area. The average effective scattering length for all 20 DOMs is $100 \pm 15 \mathrm{~cm}$, while all DOMs are covered by effective scattering lengths ranging between $75 \mathrm{~cm}$ and $150 \mathrm{~cm}$. This result agrees to the effective scattering length found by the original AMANDA analysis $(100 \mathrm{~cm}$ to $350 \mathrm{~cm})$ within the stated uncertainties.

\section{Modeling the angular acceptance}

One can employ the same Geant 4 simulation described in Sect. 3 to calculate an effective modification to the angular acceptance due to the hole ice. This can be simulated by placing a uniform beam some distance away from the DOM. As the beam is rotated around the DOM the relative photon counts between simulations with no hole ice and a given hole ice column give a relative correction factor for 
each simulated angle. Applying this correction factor to the angular acceptance measured in the lab yields the average DOM angular acceptance in the ice, assuming a homogeneous $60 \mathrm{~cm}$ diameter hole ice column. The simulation has been performed for a variety of sizes and distances of the uniform beam, as different size regions of the hole ice column are probed. The resulting correction curve was found to be stable for beams larger than $1 \mathrm{~m}$ in radius placed more than $\sim 2.5 \mathrm{~m}$ away from the DOM. Figure 5 depicts the obtained angular acceptance for the best fit effective scattering length of $100 \mathrm{~cm}$ compared to the lab measurement and the currently used default IceCube hole ice model [6]. The default IceCube hole ice model and the acceptance obtained from the presented method agree well, especially considering the very different experimental approaches and simulation assumptions.

\section{Summary and outlook}

A new experimental approach to determine the scattering properties within the refrozen IceCube drill holes has been presented. Light originating from LEDs within one DOM detected by the photomultiplier of the same DOM has been used for this measurement. The measured light intensities have been compared to a simulation assuming Henyey-Greenstein scattering in a $60 \mathrm{~cm}$ homogeneous hole ice column with the DOM situated in its center. The best fit scattering length has been used to simulate an effective modification to the angular acceptance of the IceCube DOMs, which agrees well with the currently used model $[6,10]$. In the future more complicated models, representing the structure as seen by the cameras, will be tested. These should result in a higher relative acceptance for head-on illumination, which would draw a consistent picture with recent inter-string flasher analysis.

\section{References}

[1] A. Achterberg et al., Astropart. Phys. 26, 155 (2006)

[2] R. Abbasi et al., Nucl. Instr. Meth. Phys. Res. 618, 139 (2010)

[3] T. Benson et al., Annals of Glaciology 55, 105 (2014)

[4] H. Shoji, C.C. Langway, Nature 298, 548 (1982)

[5] N.E. Bramall et al., Geophys. Res. Lett. 32 (2005)

[6] M. Aartsen et al., Nucl. Instr. Meth. Phys. Res. 711, 73 (2013)

[7] M.G. Aartsen et al., Physical Review D 91 (2015)

[8] P. Hulth (VLVNT, 2013)

[9] E. Andres et al., Astropart. Phys. 13, 1 (2000)

[10] A. Karle, K. Woschnagg (AMANDA internal, 1998)

[11] P. Niessen (AMANDA internal, 1998)

[12] D. Chirkin (IceCube internal, 2015)

[13] P. Olbrechts et al. (AMANDA internal, 2001)

[14] L. Gerhardt (IceCube internal, 2007)

[15] R. Abbasi et al., Nucl. Instr. Meth. Phys. Res. 601, 294 (2009)

[16] L.C. Henyey, J.L. Greenstein, ApJ 93, 70 (1941) 